Classification

Physics Abstracts

$07.80-10.91-13.89-61.16 \mathrm{D}$

\title{
Developments in EELS instrumentation for spectroscopy and imaging
}

\author{
Ondrej L. Krivanek, Alexander J. Gubbens and Niklas Dellby $\left(^{\star}\right)$ \\ Gatan Research and Development, 6678 Owens Drive, Pleasanton, CA 94588, U.S.A.
}

(Received February 08, 1991; accepted March 15, 1991)

\begin{abstract}
Recent developments in instrumentation for electron energy loss spectroscopy (EELS) at Gatan R\&D are reviewed. A 10-channel intrinsic Si detector with single-electron detection capability is being developed for fast energy-filtered imaging and elemental mapping in the STEM mode. A new type of an imaging filter suitable for attachment at the end of the electron-optical column of a transmission electron microscope has been designed and built. The design of the filter is described, and its electron-optical properties are compared with the properties of an optimized $\Omega$-filter. Finally, an unconventional design of an ultra-high resolution electron spectrometer which does not use any retardation and yet should be able to attain an energy resolution of a few meV at primary energies around $200 \mathrm{keV}$ is proposed.
\end{abstract}

\section{Introduction.}

Progress in electron energy loss spectroscopy (EELS) in the electron microscope now spans almost five decades since the pioneering work of Hillier and Baker [1], but the last few years have witnessed particularly rapid developments. The pursuit of high detection efficiencies through the development of parallel-detection electron energy loss spectrometers, which was initiated by Egerton [2], Johnson [3], and others, and developed into an efficient instrument by Shuman [4], has resulted in a commercially available spectrometer $[5,6]$, which is now permitting new levels of performance to be attained in many laboratories. Energy-filtered imaging, which was originally developed by Castaing and Henry [7], has also lead to a commercially available instrument, and is now being used for imaging with improved contrast as well as for chemical mapping of major constituents [8]. There is also much interest in improving the attainable energy resolution, both by optimizing the performance of spectrometers attached to microscopes using cold field emission guns with a narrow energy spread [9-11], and by decreasing the energy spread of the gun further through the use of a monochromator $[12,13]$.

This article divides the EELS instrumentation field into three categories: parallel-detection spectrometers able to form a dispersed spectrum and to detect the whole spectrum at the same time; imaging spectrometers able to form both energy-filtered images (or diffraction patterns)

$\left(^{\star}\right)$ now at: Laboratory of Nuclear Physics, MIT, Cambridge, MA 02139, U.S.A. 
and dispersed spectra; and instruments for high (energy) resolution EELS (HREELS). Developments in all three categories are reviewed, and the designs for several new EELS instruments are presented.

\section{Energy-filtered imaging in a STEM.}

The Gatan parallel-detection electron energy-loss spectrometer (PEELS) [5,6] now routinely provides spectra in which the main source of noise is the statistical fluctuation in the number of arriving electrons (i.e., the spectra are acquired with a detective quantum efficiency (DQE) greater than 0.5 ), achieves an energy resolution which is more than $90 \%$ limited by the energy spread of the electron microscope gun, and is easy to operate. It has pushed the minimum detectable mass (MDM) obtainable by EELS towards the single atom level [14], and has improved the minimum detectable mass fraction (MMF) so that it is now comparable or better than the MMF detectable by EDXS even for elements as heavy as Fe [15]. The attainable energy resolution is now $0.3-0.5 \mathrm{eV}$ on routine basis [11] when the spectrometer is mounted on a (scanning) transmission electron microscope ((S)TEM) equipped with a cold field-emission gun operating at $100 \mathrm{kV}$. At the same time, newly available computer software $[16,17]$ is making chemical microanalysis with PEELS almost as easy as microanalysis by energy-dispersive X-ray spectrocopy (EDXS).

Given the highly developed state of the PEELS when used for spectroscopy, our R\&D effort in instrumentation is now focusing on the efficient acquisition of energy-filtered images and diffraction patterns, both in scanning and fixed beam transmission electron microscopes (STEM and TEM). The instruments under development are expected to be useful both for forming elasticonly energy-filtered images and diffraction patterns that give better contrast and resolution, and are easier to interpret than unfiltered data, and for chemical mapping by acquiring and processing images formed by inner-shell loss electrons.

Before describing our work in detail, let us review the different ways in which energy filtered images can be acquired, and processed to yield spatially-resolved chemical maps of a specimen. The basic task consists of acquiring an energy loss spectrum at each point $(x, y)$ of a specimen area of interest, quantifying the spectrum from each point to obtain chemical information, and displaying the chemical information as a two-dimensional chemical map. The raw data can be shown schematically as occupying a 3-dimensional data space whose coordinates are $x, y$, and the energy loss $\Delta E$. Since we live in a 3-dimensional world in which one dimension is needed for transmitting the electron beam to the detector, we can at most detect only one 2-dimensional slice through the 3-D data space at a time. Alternately, we can explore the 3-D data space using a series of 1-dimensional vectors, and we can also explore it point by point. This last approach is that of a serial detection spectrometer acquiring a series of energy-filtered images sequentially in all the 3 dimensions, and is clearly very inefficient. Restricting ourselves to cases in which at least a one-dimensional vector is acquired at a time still leaves at least 4 possible strategies for gathering the needed data.

The different approaches are illustrated schematically in figure 1. The approach depicted in (a) is used when chemical mapping is done with a PEELS attached to a STEM instrument. The probe is positioned at each point $(x, y)$ in turn, and a whole energy loss spectrum is acquired. Methods (b) and (c) become possible if the sample is illuminated by a "line" probe which is parallel to the $y$-axis, or if a narrow slit which is parallel to the $y$-axis is inserted just in front of a spectrometer. In method (b), a 2-dimensional detector is used to acquire data in the $(y, \Delta E)$ plane. This method is especially useful for quantitative measurements of $(q, \Delta E)$ dispersion surfaces (where $q$ is the scattering vector). It has been used extensively both by Möllenstedt spectrometers [18] and Wien filters [19], with photographic film serving as the detector. Method (c) is especially suitable when 
a 1-dimensional detector is available, and it is desirable to capture one energy-filtered line profile at a time. Finally, method (d) is that of an energy-filtering transmission electron microscope (EFTEM), which collects a two-dimensional image with one energy window at a time, but has to explore different energies sequentially. (The discussion above is equally applicable to acquiring energy-filtered diffraction patterns, but for the sake of simplicity, we restricted it to the imaging case).

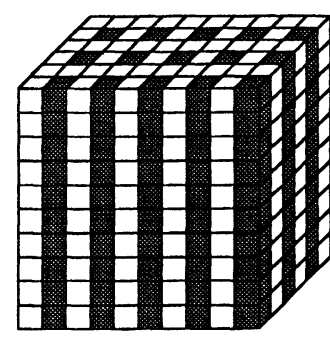

a

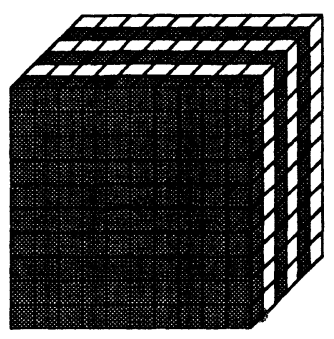

b

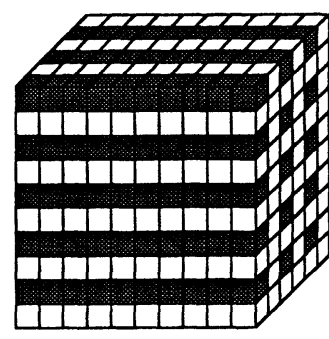

C

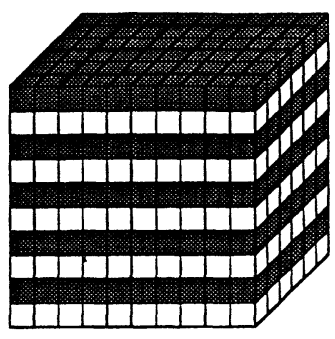

d

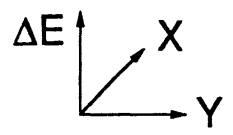

Fig. 1. - Four ways to acquire data for chemical mapping: a) PEELS + STEM producing a spectrum from each specimen point; $b$ ) a filter producing a spectrum of a line on the specimen dispersed by energy and position onto a 2-D detector; c) as (b), but using a 1-D detector; d) energy-filtering TEM (EFTEM) producing an energy-selected image.

The chief advantage of the STEM plus PEELS approach (a) is that detailed quantitative analysis can be performed at each individual point of the specimen using the recorded data, and that only one exposure is needed for acquiring the whole chemical map, thus minimizing radiation damage. However, the approach is rather slow. The read-out of a whole 1024-channel energy loss spectrum at each point typically takes a minimum of 25 msecs, resulting in a 109 minute minimum acquisition time for a $512 \times 512$ pixels map. Another disadvantage is that vast amounts of data are generated - without compression, a single $512 \times 512 \times 1024$ data set stored in floating point format occupies 1 GigaByte (1024 MBytes) of computer memory.

We are currently pursuing two approaches to overcome these difficulties. In the first approach, the standard PEELS detector is supplemented by a dark-field (DF) detector located inside the spectrometer entrance flange, and computer-controlled hardware and software which can move a STEM probe across a sample and acquire and display a STEM image. In standard operation, a DF STEM image of the specimen area of interest is first formed in a fraction of a second, and displayed on the screen of a Macintosh II which also controls the acquisition of PEELS data. Points of interest are defined in the image using the Macintosh mouse, and a reduced area or a line profile are explored in detail using the standard PEELS detector. In this way, spatially resolved chemical information can be obtained in a few seconds with an essentially unmodified PEELS.

In the second approach, we are developing a new type of a "fast" parallel detector specifically optimized for elemental mapping. The design criteria are 1) the detector should be able to detect single 100-400 keV electrons without being cooled, 2) the detector should not saturate for incident 
electron fluxes as high as a few nA (i.e., it should have a dynamic range grater than $10^{7}$ ), 3) the detector may have a smaller number of channels than the standard PEELS detector, but each channel should be individually addressable, and 4) the detector should be small enough so that it can be incorporated next to the photodiode array used in the standard PEELS. Compared to the mapping detector under development at EMBL in Heidelberg [20], our detector will be smaller, less demanding to operate, and considerably more economical.

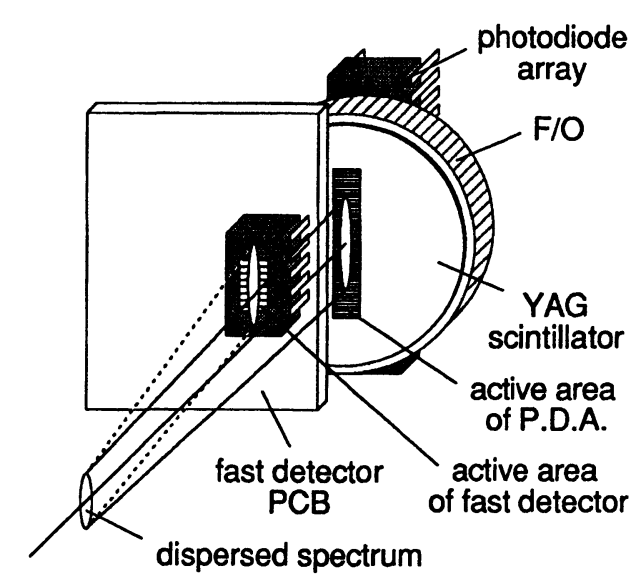

Fig. 2. - Ten-channel fast detector located next to the 1024-channel photodiode detector in the PEELS detector head.

Figure 2 shows a schematic picture of the fast detector under development. The detector is optimized for direct illumination by electrons of $80 \mathrm{keV}$ to $400 \mathrm{keV}$ in energy. It is fabricated on a $50 \mu \mathrm{m}$ thick epitaxial layer of intrinsic $\mathrm{Si}$, and consist of an array of 10 photodiodes each $3 \mathrm{~mm}$ wide by $0.25 \mathrm{~mm}$ high. It is located next to a 1024-channel photodiode array of the type used in the standard Gatan PEELS. The dispersed spectrum can be deflected transversely so that it is either detected directly by the fast detector, or projected onto the YAG scintillator which produces the light detected by the larger photodiode array. If the beam is resting on the YAG, 1024-channel spectra can be acquired as in the standard Gatan PEELS. Each channel of the fast detector corresponds to roughly 10 channels of the photodiode array, whose individual channels are $2.5 \mathrm{~mm}$ wide by $25 \mu \mathrm{m}$ high. The whole fast detector corresponds approximately to the central 100 channels of the photodiode array.

The signal from each fast detector channel is amplified by a separate preamplifier, and separated into two data paths. The first path consists of a discriminator followed by pulse counting circuitry, and is used when the signal levels are low enough to permit the counting of individual arriving electrons. The second data path consists of an analog amplifier and an A/D converter, and is used at electron arrival rates above about $1 \mathrm{MHz}$, at which point the counting chain is beginning to saturate. The data from the 10 detectors is accumulated in parallel into 10 independent registers, which are read out serially using a direct memory access (DMA) protocol. Any number of the individual detectors can be read at each image point, from a single detector being read repeatedly while the signal falling on the others is ignored, to all 10 detectors being read in turn. The design maximum read-out frequency is $1 \mathrm{MHz}$, enabling $512 \times 512$ pixel images to be read out in about 0.25 seconds when a single detector is used. 
The single detector mode will be particularly useful for zero-loss imaging in the STEM mode. For elemental mapping, all the detectors will be read at each image point. In this way, 10 energyloss images will be acquired in the vicinity of a single edge, which should make reliable extrapolation and subtraction of the background readily possible. Separate edges will require separate scans of the sample, but the increased speed made available by the fast detector will, in most cases, justify the increase in the required electron dose.

First experiments with the new detector confirmed that a directly illuminated intrinsic Si detector is able to convert each arriving $100 \mathrm{keV}$ electron into a recognizable pulse whose magnitude is significantly higher than the background noise (Fig. 3), even though the detector is not cooled. We are now eliminating teething problems in the signal-processing electronics associated with cross-talk between the 10 channels of the detector, and writing the software that will support all the possible operating modes of the fast detector. We anticipate that chemical mapping of most elements with the fast detector will offer sensitivity and acquisition times considerably better than those possible when acquiring elemental maps using EDXS.

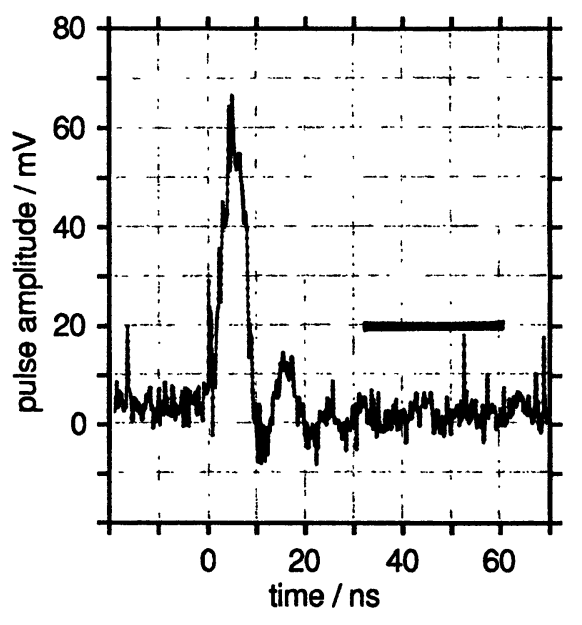

Fig. 3. - A pulse due to the arrival of one $100 \mathrm{keV}$ electron into one channel of the fast detector. The horizontal bar marks the maximum height of pulses due to background noise. The narrow spikes are due to the sampling noise of the digital oscilloscope used.

In a separate project, we are also exploring the approaches to energy-filtered imaging depicted by figures $1 \mathrm{~b}$ and $\mathrm{c}$ through relatively simple modifications of the existing PEELS. For these cases, no energy selecting slit is required, since a focussed spectrum is formed only at the final detector. The spectrum needs to be spread in the non-dispersion direction $y$ in addition to energy, but this is easily achieved by modifying the quadrupole excitations of an otherwise standard PEELS. A slit aperture is inserted in front of the PEELS to probe only one line parallel to the $y$-axis at a time, and the one-dimensional detector of the standard PEELS is either replaced by a two-dimensional detector to obtain regime 1 (b), or is made rotatable by $90^{\circ}$ to obtain regime 1 (c). A two-orthree-dimensional energy-filtered image is then built up by scanning the non-filtered image (or diffraction pattern) across the entrance slit (i.e., in the $x$-direction), and recording a $(y, \Delta E)$ map for every position $x$. A PEELS using a rotatable one-dimensional detector has been constructed, and is presently being tested for the acquisition of energy-filtered diffraction patterns $[21,22]$. 


\section{Energy-filtered imaging in a TEM.}

The acquisition of energy filtered images in a TEM, as illustrated by figure 1d, requires an instrument consisting of two separate stages. In the first stage, an image or a diffraction pattern of a specimen is transformed into an electron energy loss spectrum, and a suitable energy interval is selected by an energy-selecting slit. In the second stage, the spectrum is transformed back into an image which should show no dependence on the electron energy, and be free of bluring and geometric distortion.

The first high-quality energy-filtered images were produced by Castaing and Henry [7] using a filter which sent the electron beam through a $90^{\circ}$ electromagnetic sector, reflected the beam from an electrostatic mirror, and then sent the beam through the electromagnetic sector for a second time. The overall system had two mirror planes of symmetry (one at right angles to the original beam direction, the other containing the central beam trajectory). This minimized several geometric distortion terms, and caused the beam to emerge along the same line as it went in, thereby permitting an in-line design of the overall instrument.

The use of the electrostatic mirror in the Castaing-Henry design makes this type of imaging filter unsuitable for primary beam voltages greater than about $100 \mathrm{kV}$. At voltages of $200 \mathrm{kV}$ and greater, a more suitable imaging filter is therefore the electromagnetic-only $\Omega$-filter introduced by Senoussi et al. [23], further developed by Zanchi et al. [24], and optimized by Rose and coworkers $[25,26]$.

The Castaing-Henry and the $\Omega$-filters both have the disadvantage that they must be incorporated inside the imaging part of a microscope column, which makes it difficult to retrofit them to existing microscopes. From this point of view, an imaging filter which attaches to the bottom of an electron microscope just like a simple magnetic spectrometer would be highly preferable. Several researchers have investigated this possibility. Shuman used a parallel-detection spectrometer employing a single magnetic sector followed by two round lenses, and a two-dimensional SIT camera. By adding an energy-selecting slit in front of the two round lenses and changing the lens excitation, he was able to produce two-dimensional energy-filtered images in which the energy dispersion was canceled to first order [27]. Ajika et al. added a drift tube without any lenses behind the energy-selecting slit of the Gatan 607 spectrometer, and mounted a TV camera at the end of the drift tube [28]. Energy dispersion was not canceled in their images, which were also heavily distorted. However, they used a narrow energy window, compensated for the distortion by stretching the images as needed using a computer, and were able to record energy-filtered lattice images of silicon. Ahn and Krivanek used a single magnetic sector spectrometer with an energyselecting slit, three quadrupole lenses after the slit, and a TV camera [29,30]. They were able to cancel the energy dispersion to first order, and produced sharp and relatively undistorted energy filtered lattice images of $\mathrm{Si}$, and chemical maps of a $\mathrm{Cr}$-C layer composite. More recently, McMullan et al. used a similar approach, and obtained energy-filtered diffraction patterns with a parallel detection spectrometer which included an energy-selecting slit placed in front of 4 quadrupole lenses, with the whole system mounted on a VG HB501 STEM [31].

None of the above approaches, however, addressed the problem of aberrations and distortions in the energy filtered images beyond the first order. This was tolerable as long as the images produced were not more than about $256 \times 256$ independent pixels in size. (An image can be detected with a sensor which has a large number of pixels, but if each image point is blurred so that it extends over several pixels, the image size expressed in independent pixels will be much smaller). For larger images, the simple optics was not able to cope, and image aberrations became unacceptable.

The first, spectrum-producing stage of the filter should satisfy three first-order and three secondorder constraints, which are best explained using the $x, y, z, x^{\prime}, y^{\prime}, E$ coordinate system and nota- 
tion ([32], see also Fig. 5). In this system, $x$ and $y$ measure the distance of a beam perpendicular to the optic axis, which curves as it goes through the magnetic sector, and $z$ is the distance along the axis. $x^{\prime}$ and $y^{\prime}$ stand for $\mathrm{d} x / \mathrm{d} z$ and $\mathrm{d} y / \mathrm{d} z$ respectively, and measure the angle a beam makes to the axis. $\mathrm{d} E$ measures the difference in energy of the beam under consideration from the reference beam which travels down the optic axis. The origin of the coordinate system is most conveniently placed in the entrance object plane of the spectrometer containing a crossover of limited spatial extent $(\Delta x, \Delta y)$, but considerable angular spread $\left(\Delta x^{\prime}, \Delta y^{\prime}\right)$. Such a small crossover exists under all standard operating conditions of a TEM in the back focal plane of the projector lens. Its existence is due to the fact that the angular spread produced by the projector lens is always large (so as to illuminate a large portion of the viewing screen), and the reciprocal relationship between the angular spread of a beam passing through a crossover and the size of the crossover.

In an imaging filter using the projector lens crossover as the entrance object, the first-order constraints of the first, spectrum-producing stage are:

$$
\mathrm{d} x / \mathrm{d} x^{\prime}=0, \quad \mathrm{~d} y / \mathrm{d} y^{\prime}=0, \quad \text { and } \quad \mathrm{d} x / \mathrm{d} E=\text { as high as poss. }
$$

The first two constraints specify that the spectrum should be focussed in both the $x$ and the $y$ directions, and the third constraint specifies that the spectrum should have as high an energy dispersion as possible. The focus in $y$ is not strictly necessary, but is highly useful because a doubly focussed spectrum passes through a very small segment of the energy-selecting slit, which decreases the mechanical precision required of the slit to easily attainable levels.

The second-order constraints of the first stage are:

$$
\mathrm{d}^{2} x / \mathrm{d} x^{\prime 2}=0, \quad \mathrm{~d}^{2} x / \mathrm{d} y^{\prime 2}=0, \quad \text { and } \quad \mathrm{d}^{2} x / \mathrm{d} x^{\prime} \mathrm{d} E=0 .
$$

Here, the first two constraints specify that the spectrum should be focussed in the $x$ direction to second order, and are important for obtaining a good energy resolution even when a beam of large angular spread is accepted into the spectrometer. The third constraint specifies that the plane of the focussed spectrum should be perpendicular to the optic axis, and ensures that when the imaging filter is operated as a parallel-detection spectrometer, the whole spectrum will be detected in focus. In a filter using a single magnetic sector to disperse the beam into an energy spectrum, these three constraints can be satisfied by choosing the appropriate entrance and exit face angles and curvatures of the magnetic sector. Several other second-order constraints are made redundant by reasons of symmetry and the small size of the entrance object.

The requirements of the second, image-producing stage of the filter are more comprehensive. This increase in complexity is due to the fact that in the image plane of an imaging filter, the properties in both $x$ and $y$ should be controlled to second order. Assuming again that the spectrometer uses as its entrance object a crossover whose spatial extent can be ignored for the moment, the first-order constraints for the second stage are:

$$
\mathrm{d} x / \mathrm{d} x^{\prime}=\mathrm{d} y / \mathrm{d} y^{\prime}=M L \quad \text { and } \quad \mathrm{d} x / \mathrm{d} E=0,
$$

where $M$ is the magnification of the image projected onto the detector relative to the image that would be seen on the viewing screen if the screen were lowered, and $L$ is the distance of the lowered screen from the projector lens crossover.

The first two constraints specify that the magnification of the image in the dispersion $(x)$ and the non-dispersion ( $y$ ) directions should be the same, and should be adjustable to suit the detector employed. The third constraint specifies that the image should show no first-order dependence on electron energy. These three constraints can be satisfied with just three quadrupole lenses, as was demonstrated with practical examples by Ahn and Krivanek $[29,30]$. Note that other necessary 
constraints such as $\mathrm{d} y / \mathrm{d} E=0$ are automatically satisfied due to the mirror symmetry about the $y=0$ plane, and they have therefore not been listed here.

The second-order constraints for the second stage are:

$$
\begin{aligned}
& \mathrm{d}^{2} x / \mathrm{d} x^{\prime 2}=0, \quad \mathrm{~d}^{2} x / \mathrm{d} y^{\prime 2}=0, \quad \mathrm{~d}^{2} y / \mathrm{d} x^{\prime} \mathrm{d} y^{\prime}=0, \\
& \mathrm{~d}^{2} x / \mathrm{d} x^{\prime} \mathrm{d} E=0, \quad \mathrm{~d}^{2} y / \mathrm{d} y^{\prime} \mathrm{d} E=0, \quad \text { and } \quad \mathrm{d}^{2} x / \mathrm{d} E^{2}=0 .
\end{aligned}
$$

Note that because each constraint listed above applies to the plane of the detector, it is different from an identical-looking constraint applicable to the plane of the energy-selecting slit. Other second-order constraints are again made redundant by symmetry and the small size of the entrance object.

The top three constraints banish the purely geometric second-order image distortions not canceled by the single mirror symmetry of the system. These distortions are illustrated schematically in the top row of figure 4 . The bottom three constraints banish distortions of the image which depend either wholly or partly on the electron energy, and are illustrated in the bottom row of figure 4 .
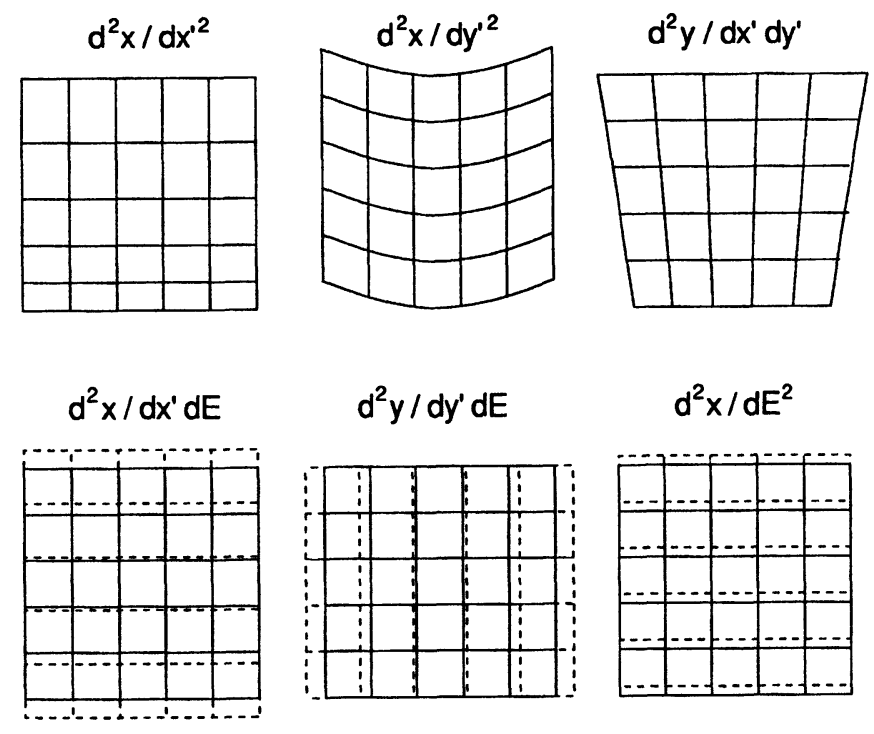

image due to electrons with energy $E$ image due to electrons with energy $E-\Delta E$

Fig. 4. - Second order aberration terms affecting the quality of energy filtered images produced by a filter focussing on a beam crossover of negligible size. The schematic "test object" is a grid of $5 \times 5$ identical squares, located some distance away from the crossover. The distorted images show how the various aberrations influence the image. Top row: geometric aberrations which cause distortion in the filtered image. Bottom row: chromatic aberrations which cause image blurring for non-zero width of the energy window.

The magnitude of the second-order geometric distortion terms in an imaging filter based on a single magnetic sector but not corrected to second order is such that the geometric distortions in an image whose detector spans $256 \times 256$ pixels amounts to about 1 percent, and to $10-20$ percent 
in an image projected onto a $1024 \times 1024$ pixel detector. These distortions could, in principle, be removed by a computer program which remaps the image onto a net which has been stretched to compensate for the distortion. However, the computer would not be able to compensate for the energy-dependent terms, which will cause image blurring whose magnitude will depend both on the size of the image field, and on the width of the selected energy window. For a 1024 by 1024 pixel detector, the widest energy window that could be admitted without noticeable blurring would be about $5 \mathrm{eV}$ large at $200 \mathrm{keV}$. This would be tolerable for purely elastic imaging, but would be highly unsatisfactory for chemical mapping, for which energy windows up to $100 \mathrm{eV}$ wide may be required. We therefore decided to investigate whether all the important secondorder terms could be corrected electron-optically in a magnetic filter which attaches at the end of the microscope column. Our design [33] takes into account the fact that the filter's detector is likely to be located a considerable distance from the vertical axis of the microscope, which makes using a photographic film camera impractical. Instead of aiming for an image field of $10^{4}$ by $10^{4}$ pixels, as would be necessary if photographic film were to be used, our design therefore aims to produce images suitable for detection by a solid state electronic sensor with an active area of about $2 \mathrm{~cm} \times 2 \mathrm{~cm}$ in size, consisting of $1024 \times 1024$ pixels. Because it will make the filtered images immediately available for display on a computer screen, and will also make it possible to obtain background-subtracted chemical maps on-line, such a sensor will in any case be preferable to photographic film.

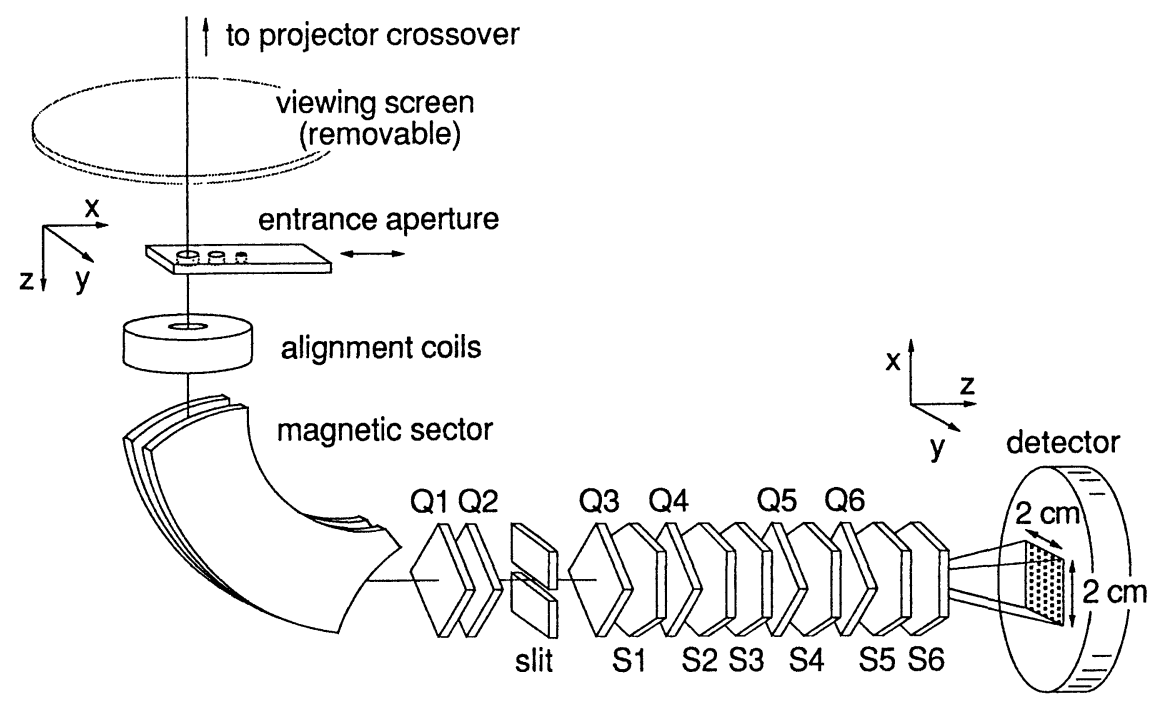

Fig. 5. - Schematic diagram of the imaging filter under development. Note the axes marking the coordinate system.

The principal elements of our design are shown schematically in figure 5. A single magnetic sector of $10 \mathrm{~cm}$ bending radius is preceded by focussing quadrupoles, sextupoles and alignment dipoles similar to those used by the PEELS [5], and followed by an energy-selecting slit, 6 quadrupole and 6 sextupole lenses, and a 2-dimensional slow-scan CCD camera [34], which is able to deliver images whose dynamic range, sensitivity, and linearity exceed those normally attainable by photographic film. Two of the post-sector quadrupoles are situated in front of the energy-selecting slit and therefore belong to the first, spectrum-producing stage of the filter. They increase the 
dispersion of the magnetic sector by about $5 \mathrm{x}$ from $1.8 \mu \mathrm{m} / \mathrm{eV}$ at $100 \mathrm{keV}$ primary energy (and $0.4 \mu \mathrm{m} / \mathrm{eV}$ at $400 \mathrm{keV})$ to about $10 \mu \mathrm{m} / \mathrm{eV}(2 \mu \mathrm{m} / \mathrm{eV}$ at $400 \mathrm{keV})$. At the same time, they make it possible to fine-tune the double focussing of the spectrum at the slit.

The four quadrupoles situated after the slit can be operated in two different modes. In the first mode, the quadrupoles project a focussed image of the spectrum formed at the slit onto the 2-dimensional detector, with a magnification which can be varied independently in the $x$ and the $y$ directions (as in a standard PEELS). In this mode, the slit is either open wide to permit the observation of a whole spectrum, or it is closed down to allows the precise selection of an energy window of interest by direct observation of the closed-down slit and the energy window within it. In the second mode, the quadrupoles cancel the energy dispersion produced by the magnetic sector, and project a magnified image of the specimen satisfying the three first-order constraints of equation (3) onto the $2-\mathrm{D}$ detector. The image is typically magnified about $15 \mathrm{x}$ with respect to the image that would appear on the viewing screen of the microscope if the screen were lowered, and a final image with a magnification of $300000 x$ therefore needs the microscope to produce a magnification of only $20000 x$ on the viewing screen.

The optimum operation of our filter thus requires that the projector lens be weakened by about a factor of 15 relative to its normal condition. Instead of increasing the total magnification by about $150 \mathrm{x}$ and illuminating the whole viewing screen as in a normal TEM, the projector lens should increase the magnification by only about 10 times, and should illuminate only the central $3 \mathrm{~mm}$ or so of the viewing screen. As a result, the quality of the shielding against stray electromagnetic fields in the viewing chamber must be similar to the shielding higher up in the electron microscope column. This is because movement of the image by as little as $2 \mu \mathrm{m}$ at the level of the viewing screen will cause a noticeable blurring of the final image. In practice, it means that the viewing chamber needs to be made out of soft steel rather than brass.

Electron trajectories through the filter operating in the image (as opposed to spectroscopy) mode are shown schematically in figure 6. Canceling the energy dispersion in the final image requires that the quadrupoles run with different polarities, and results in a beam path through the apparatus that contains one crossover in the $x$-direction (at the slit), and four crossovers in the $y$-direction. The energy dispersion along the beam path varies widely, from zero in front of the sector to a maximum situated just after the energy-selecting slit, and back to zero at the detector. This means that the beam inside the 6 sextupoles placed along the beam path has very different first-order properties. This is highly desirable if the second order properties of the filter are to be optimized. For instance, energy dispersion $(\mathrm{d} x / \mathrm{d} E)$ is high inside sextupoles $\mathrm{S} 1$ to $\mathrm{S} 3$, but much smaller inside sextupoles S4 to S6. This means that when sextupoles S1 to S3 are excited, they change the three energy-dependent terms of equation (4b), whereas sextupoles S4 to S6 have little effect on the chromatic aberrations. Hence sextupoles S1 to S3 can be used to correct the chromatic aberrations, and sextupoles S4 and S6 can be used to correct the geometric distortions of (4a), without changing the energy-dependent terms. As another example, a $y$-crossover in the beam occurs near sextupoles S2 and S5, which means that these sextupoles only affect those terms of equation (4) which are dependent on parameters other than $y^{\prime}$. Thus sextupole S5 mostly affects only the first term of $(4 \mathrm{a}), \mathrm{d}^{2} x / \mathrm{d} x^{\prime 2}$. Similarly, other sextupoles have a strong effect on only one or at most two terms listed in equation (4). A unique combination of sextupole currents then leads to a condition where all the 6 terms of (4) are simultaneously forced to zero, and the filter is fully second-order corrected.

The validity of the general ideas described above was confirmed by computer simulations of both the imaging and the spectroscopy modes. The simulations were valid to second order, and took account of all the geometric as well as all the chromatic terms. They showed that a full correction of the second order terms can be obtained with quadrupole and sextupole excitations which are easily attainable in practice. A filter based on the calculated solution was then built, in 


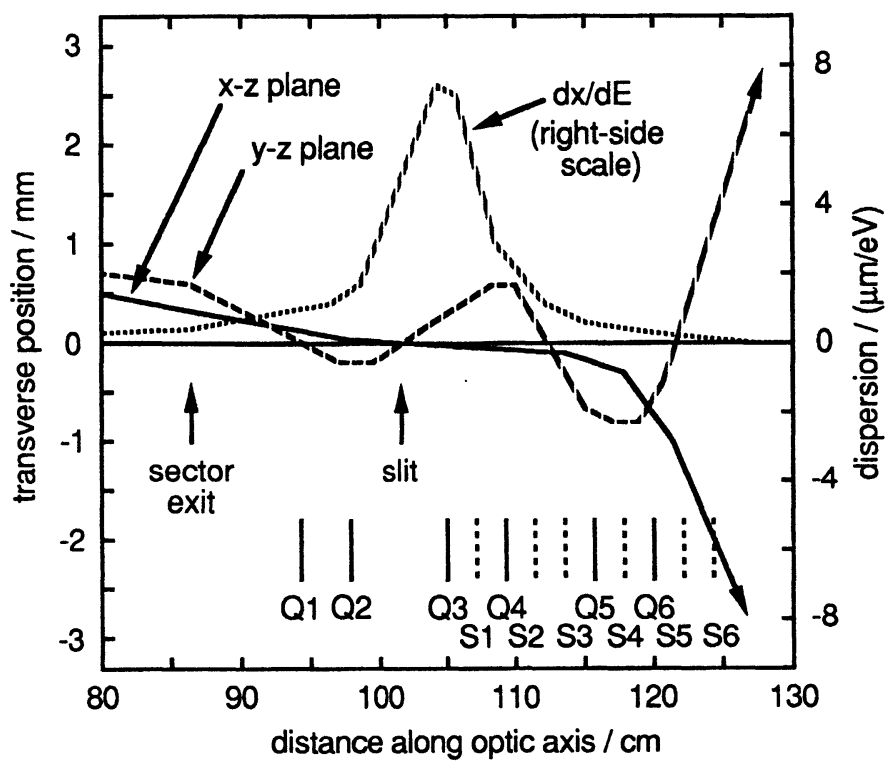

Fig. 6. - Schematic trajectories of beams traveling in the $x-z$ and $y-z$ planes through the filter when operating in the imaging made, and the energy dispersion $(\mathrm{d} x / \mathrm{d} E)$. The trajectories apply to beams heading for the edges of a $2 \mathrm{~cm}$ by $2 \mathrm{~cm}$ detector situated at $z=140 \mathrm{~cm}$. The energy dispersion is shown for a primary voltage of $200 \mathrm{kV}$. The locations of the principal optical elements are marked.

order to test the validity of the calculations, and to see whether there are any serious problems due to misalignment of the many optical elements required.

The first tests of the electron-optical performance of the filter at $100 \mathrm{keV}$ primary energy are presented in figure 7, which shows the image of a test object created by placing a 9-hole aperture into the entrance image plane of the spectrometer, where it simulates the specimen image that would be normally present in this plane. Image $7 \mathrm{a}$ was obtained with only the quadrupoles excited. The original 9 holes were situated on a square grid, and the deviations of their images from the corresponding positions illustrate the presence of geometric distortions. The most visible distortion is due to the term $\mathrm{d}^{2} x / \mathrm{d} x^{\prime 2}$. It shows up as a difference in the space between the top and the middle rows of dots, compared to the space between the middle and the bottom rows. Image $7 \mathrm{~b}$ was obtained under the same condition as (a), but with a square-wave modulation of $\pm 50 \mathrm{~V}$ applied to the drift tube leading through the magnetic sector. The modulation simulated imaging with an energy window $100 \mathrm{eV}$ wide. The fact that the central disk remains sharp proves that first-order image dependence on energy can be canceled by the quadrupoles alone. However, the disks in the outer columns are badly blurred (because $\mathrm{d}^{2} y / \mathrm{d} y^{\prime} \mathrm{d} E$ has not been corrected), and this shows that the cancellation of the second order terms dependent on energy requires the sextupoles. Image $7 \mathrm{c}$ was obtained with the sextupoles excited in addition to the quadrupoles, and with the modulation on. All the spots are now sharp and placed nearly correctly on the square grid. This shows that all the important second-order aberrations in the image plane have indeed been corrected, as predicted by the computer simulations. The remaining distortions are mostly due to the mechanical imprecision of the 9-hole aperture, which was made by drilling.

A disavantage of our design is that the required sextupole currents depend on the first-order beam trajectories, and therefore change whenever the current in any of the quadrupoles has changed. Since the full second order adjustement requires all the 6 sextupoles to be at their 


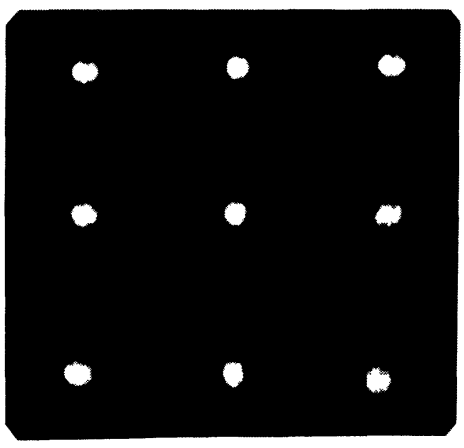

a

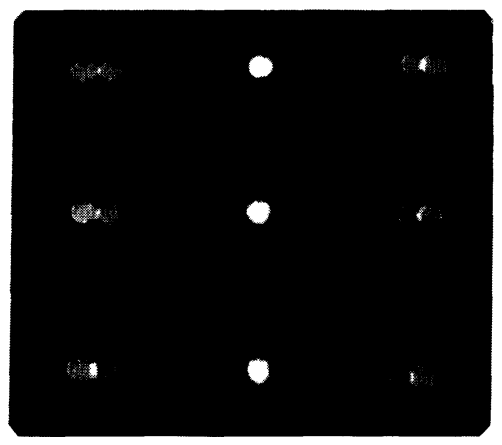

b

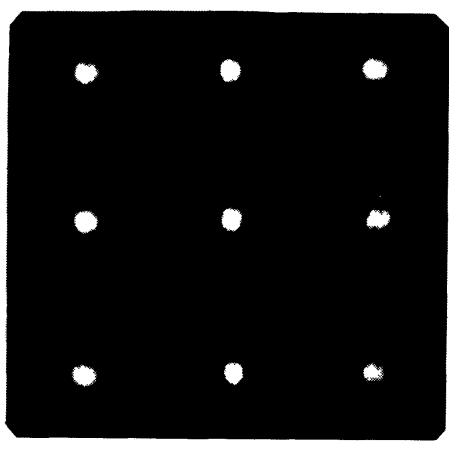

C

Fig. 7. - Test images of a 9-hole mask obtained with the filter; a) sextupoles switched off, monochromatic electron beam; b) sextupoles off, $\pm 50 \mathrm{~V}$ modulation applied to spectrometer drift tube; c) sextupoles on, $\pm 50 \mathrm{~V}$ modulation on.

correct excitations, setting up the filter in practice could be very tedious. To spare the user from having to adjust a set of 6 or more non-orthogonal controls to obtain a corrected image, we are implementing full alignment under computer control. The alignment will proceed as follows: 1 ) the sextupole currents will be set to their previous values, or to zero if the system is badly out of alignment; 2) the quadrupole currents required for the desired first-order properties will be set; 3 ) the entrance aperture of the filter will be uniformly illuminated, and the 9-hole test mask will be inserted into the plane of the entrance aperture; 4) the complete second-order aberration properties of the filter will be determined using the 9-hole pattern to characterize the geometric image distortions, and changing the high voltage of the microscope slightly to characterize the energy-dependent terms; 5) the influence of each sextupole on the aberrations will be characterized by changing the excitation of each sextupole in turn, and having the computer re-evaluate the modified aberrations; 6 ) the optimum set of sextupole excitations will be found by the computer through solving a set of 6 coupled linear equations relating the sextupole currents to the 6 second-order aberration terms; 7) the sextupole currents will be changed to the new values, and the experimentally attained aberrations will be reevaluated; 8) steps 4 to 7 will be repeated until the required precision is reached. It is estimated that the whole procedure will take about a couple of minutes, and that it will typically only have to be performed if the filter was switched off and on.

While the software that will carry out the above procedure is being developed, the filter is being tested on first applications to see how it will perform in practice. These application examples will be described in a forthcoming publication [35].

\section{Comparison of the single-sector filter with an $\Omega$-filter.}

Because our filter is markedly different from the $\Omega$-filter concept but appears to achieve comparable results, we made a detailed comparison of the electron-optical performance of the two types of filters. To evaluate the properties of both designs quantitatively, we calculated, using the same approach as for our filter, the electron optical performance of an optimized $\Omega$-filter designed by Lanio [25]. This filter consists of four magnetic sectors of $3.6 \mathrm{~cm}$ bending radius and $104^{\circ}$ bending angle, and a sextupole placed at the mid-plane of the filter. The edges of the sectors are straight to facilitate alignment, even though this means that second order aberrations in the spectrum plane 
have not been eliminated. For the purposes of the comparison, we took both filters to be operating at $200 \mathrm{kV}$ primary voltage, and producing an image of the specimen with a total magnification of $150000 x$ on the detector.

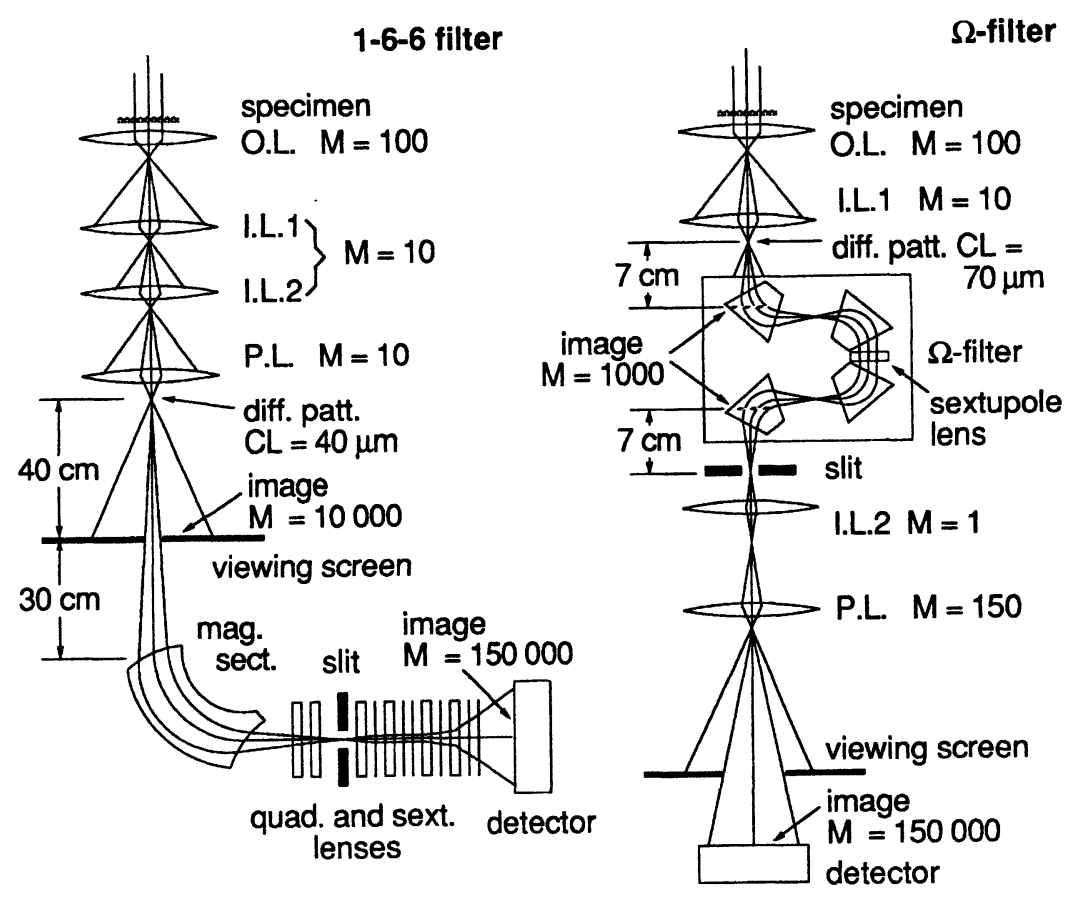

Fig. 8. - Coupling of the present filter to the electron-optical column of a TEM, compared to the coupling of an $\Omega$-filter. The principal planes, and the magnification of each component necessary to achieve a total magnification of $150000 \mathrm{x}$ are also shown. Note that a simple change in the excitation of the first intermediate lens will replace the diffraction pattern in the entrance object plane of either filter by an image of the specimen, and replace the final image by an energy-filtered diffraction pattern. This can be accomplished in either design without any other changes in the filter operating conditions.

Figure 8 compares the placement of the two filters relative to the rest of the electron microscope column. It also shows the location of the principal planes of the filters, and the magnification of the images (or diffraction patterns) located in these planes. The $\Omega$-filter is placed higher up in the imaging column of a microscope, and operates with an image whose magnification is about 10 times smaller than the magnification needed by our design. The $\Omega$-filter has short entrance and exit focussing distances, which make it well suited for placement in the middle of the column. Our filter has a particularly long entrance distance, which makes it well suited for placement at the end of the column, below the projector chamber.

Table I compares the electron-optical performance of the two filters. The table shows that the performance of the two designs is broadly comparable, but that there are also some important differences. The modest energy dispersion of the $\Omega$-filter (which is similar to the dispersion of our magnetic sector unaided by any post-sector quadrupoles), combined with the relatively large diffraction pattern which is used as its entrance object, means that the acceptance angle (measured at the specimen) into the $\Omega$-filter must be considerably restricted for obtaining good energy reso- 
lution. By comparison, the maximum acceptance angle into our filter for a comparable coupling arrangement and the same energy resolution is nearly 10 times larger. The size of the specimen area that can be imaged while maintaining a very good energy resolution is about the same for both filters. The result is that the "luminosity" (product of the largest acceptance angle times the largest image area permissible at a particular energy resolution) of our design is nearly 100 times larger than for the Lanio design. This means that considerably more signal can be gathered into our filter, and that our design will also permit more flexibility in selecting the precise operating regime.

Table I. - Comparison of the present filter, which uses a single magnetic sector, 6 post-sector quandrupoles, and 6 post-sector sextupoles, with an $\Omega$-filter using 4 magnetic sectors and 1 sextupole as designed by Lanio. For the purpose of this comparison, both filters are assumed to be working at $200 \mathrm{kV}$ primary voltage, and to be producing an image with a magnification of $150000 \times$ at the detector.

\begin{tabular}{|c|c|c|}
\hline parameter & 1-6-6 filter & $\boldsymbol{\Omega}$-filter \\
\hline $\begin{array}{l}\text { 1) entrance object in "normal } \\
\text { operation" (image at detector, } \\
\text { with magnification of } 150000 \mathrm{x} \text { ) }\end{array}$ & $\begin{array}{l}\text { diffraction pattern } \\
\text { (in back-focal plane } \\
\text { of projector lens) } \\
\text { CL }=40 \mu \mathrm{m}\end{array}$ & $\begin{array}{l}\text { diffraction pattern } \\
\text { (in back-focal plane } \\
\text { of intermediate lens) } \\
\mathrm{CL}=70 \mu \mathrm{m}\end{array}$ \\
\hline 2) magnification of ent. obj. at slit & -1.54 & 1 \\
\hline 3) energy dispersion at $200 \mathrm{kV}$ & $4.8 \mu \mathrm{m} / \mathrm{eV}$ & $.7 \mu \mathrm{m} / \mathrm{eV}$ \\
\hline $\begin{array}{l}\text { 4) largest acceptance half- } \\
\text { angle for } 1 \mathrm{eV} \text { resolution } \\
\text { (at specimen) }\end{array}$ & $39 \mathrm{mrad}$ & $5 \mathrm{mrad}$ \\
\hline 5) entrance image location & $40 \mathrm{~cm}$ below ent. obj. & $7 \mathrm{~cm}$ below ent. obj. \\
\hline $\begin{array}{l}\text { 6) image magnification } \\
\text { specimen-to-ent. im. }\end{array}$ & 10000 & 1000 \\
\hline 7) exit image location & at detector & $7 \mathrm{~cm}$ above slit \\
\hline $\begin{array}{l}\text { 8) image magnification } \\
\text { ent. im.-to-exit im. }\end{array}$ & 15 & 1 \\
\hline $\begin{array}{l}\text { 9) largest image area } \\
\text { (on the specimen) } \\
\text { for } 1 \mathrm{eV} \text { energy window }\end{array}$ & $\begin{array}{l}\text { limited by } 3 \text {-rd order } \\
\text { aberrations to about } \\
0.3 \mu \mathrm{m} \emptyset\end{array}$ & $\begin{array}{l}\text { limited by } \mathrm{d}^{2} x / \mathrm{d} x^{2} \\
\text { and } \mathrm{d}^{2} x / \mathrm{d} y^{\prime 2} \text { to } \\
0.27 \mu \mathrm{m} \emptyset\end{array}$ \\
\hline $\begin{array}{l}\text { 10) geometric distortions } \\
\text { present in image }\end{array}$ & $\begin{array}{l}\text { corrected to second } \\
\text { order }\end{array}$ & none \\
\hline $\begin{array}{l}\text { 11) maximum energy window } \\
\text { for } 1 \mathrm{k} \times 1 \mathrm{k} \text { independent } \\
\text { image points }\end{array}$ & $\begin{array}{l}\text { limited by 3-rd order } \\
\text { aberr. to about } 100 \mathrm{eV}\end{array}$ & $20 \mathrm{eV}$ \\
\hline
\end{tabular}

The imaging properties of the two filters are roughly comparable. It needs to be noted that the $\Omega$-filter attains distortion-free imaging by its elegant symmetry, whereas our design needs to use the sextupoles to achieve the same result. However, an advantage of our design is that it corrects the chromatic aberration terms shown in figure 4. In the Lanio design, these terms are small, 
but not negligible. The practical result is that even with a detector of only $1000 \times 1000$ pixels, the $\Omega$-filter will be limited to energy windows smaller than about $20 \mathrm{eV}$ (at a primary voltage of $200 \mathrm{kV}$ ) it noticeable bluring at the edges of the image field is to be avoided. At a primary voltage of $100 \mathrm{kV}$ and with 10000 by 10000 image points, the energy window would have to be smaller than $2 \mathrm{eV}$ for the $\Omega$-filter, which would be difficult to achieve even for elastic-only imaging, and not at all useful for chemical mapping.

To summarize, the electron-optical performance of the two designs is broadly comparable. Each one is particularly well adapted for its envisaged location relative to the rest of the electronoptical column. With a $1000 \times 1000$ pixel image sensor at $200 \mathrm{kV}$, the only noticeable difference between the two filters will probably be a loss of energy resolution for larger entrance angles, and a slight loss of image resolution for large energy windows in the $\Omega$-filter. The practical factors such as the ease and reproducibility of alignment and the compatibility with the normal operating modes of the electron microscope will no doubt also be important, but these need to be evaluated through practical experience.

\section{High energy-resolution EELS.}

The intrinsic energy resolution of several electron energy loss spectrometers used on $100 \mathrm{kV}$ (S)TEMs is now smaller than the $0.3 \mathrm{eV}$ energy spread of a cold field emission gun (FEG). This means that the next step in spectrometers designed for high energy resolution EELS must be to monochromate the electron beam before it reaches the specimen, and to use an energy analyzer of relatively high dispersion. The resultant improvement in energy resolution should make EELS in an electron microscope into an exciting probe of local band structure, and possibly even local vibrational modes.

To attain an energy resolution approaching a few meV, the monochromator and the energy analyzer will have to be linked in such a way that variations of a few $\mathrm{eV}$ in the primary energy will not degrade the overall energy resolution. One possible way to accomplish this is to use Wien filters for both the monochromator and the analyzer, and to deaccelerate the electron beam to an energy of a few eV inside each filter. If the same high tension (HT) supply is used for the electron gun as well as for all the deaccelerators and accelerators in such an instrument, fluctuations of the HT of several volts have no effect on the resolution, which is instead determined by the stability of the auxiliary power supply which controls the much smaller potential difference between the primary voltage and the deaccelerating voltage applied to the two filters. An instrument of this type was developed by Boersch, Geiger and coworkers, who succeeded in obtaining energy resolution better than $3 \mathrm{meV}$ already some time ago $[12,36]$ at $30 \mathrm{kV}$ primary voltage. Their approach is now being applied at $80 \mathrm{kV}$ primary voltage [13]. However, the required electrostatic deaccelerators and accelerators are not easily realizable for primary energies of $200 \mathrm{keV}$ and above, which are desirable for smaller probe size and greater specimen penetration.

Figure 9 shows an alternate arrangement [37] which should also permit an energy resolution of a few meV to be attained at a primary voltage of $100 \mathrm{kV}$ or higher, but which utilizes magnetic elements only. Four-sector $\Omega$ filters followed by magnifying quadrupole lenses are used for both the monochromator and the analyzer. The $\Omega$-filters are especially suitable because they permit an in-line design of the overall instrument, and preserve the round symmetry of the entering beam. However, other magnetic filters such as the alpha filter, or single magnetic sectors followed by magnifying quadrupoles, could be used without affecting the principle of the apparatus.

The energy selection in the monochromator is achieved by a mechanical slit. The two blades of the slit are electrically isolated, and the current falling on one blade is sensed and compared to the current falling on the other. A stabilization scheme utilizing either a feed-back to the high voltage, 


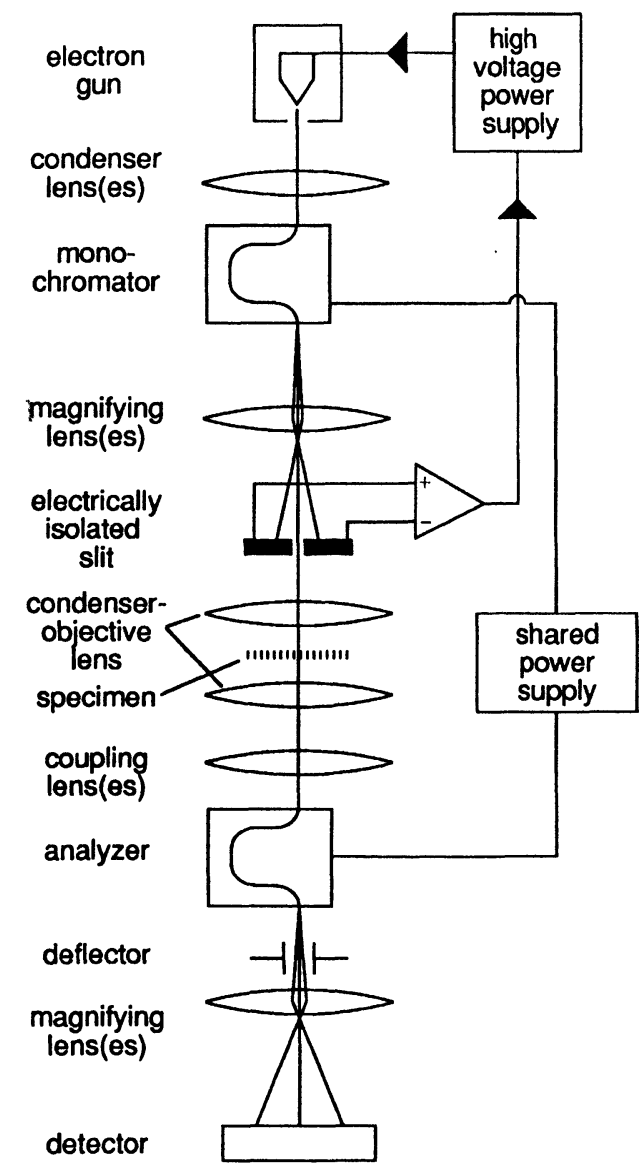

Fig. 9. - Schematic diagram of a non-deaccelerating EELS apparatus which should be capable of attaining an energy resolution of a few meV at primary energies of $200 \mathrm{keV}$ and higher.

as shown in the figure, or some other means (such as energizing an electrostatically isolated drift tube leading through the monochromator), is used to keep the beam precisely centered on the slit. The sensitivity of the feed-back scheme can be improved by making the two blades of the slit out of a scintillating material, detecting the light generated by the two slit blades by two photomultiplier tubes (PMTs), and using the output of the PMTs to monitor and stabilize the position of the beam on the slit.

A crucial element of the design is that the two magnetic filters are connected in series, i.e., that precisely the same current passes through both of them. This ensures that the energy selected by the filters is exactly equivalent at all times, and guarantees that instabilities in the high voltage or the filter current do not result in a loss of energy resolution. A small energy offset of the spectrum can be applied by energizing a weak auxiliary deflecting element (such as a weak coil) in the analyzer. Lastly, the analyzer is followed by an assembly of lenses which magnify the spectrum dispersion as high as necessary, and project it onto a parallel detector.

In order to obtain energy resolution in the meV range, the apparatus will have to be well shielded against stray magnetic fields in the central section between the energy selecting slit and the magnifying lenses following the analyzer, since stray fields penetrating into this region would 
destroy the exact correspondence between the energy selected by the monochromator and the energy selected by the analyzer. The dispersion achieved by the monochromator and the analyzer will have to be of the order of tens or even hundreds of millimeters per eV. Fortunately, this does not present any fundamental difficulties - for example, the single magnetic sector followed by 4 quadrupoles in the standard Gatan PEELS can already achieve a dispersion of $5 \mathrm{~mm} / \mathrm{eV}$ (at $100 \mathrm{keV}$ ). The stability of the power supplies which are outside the stabilization loop, such as the power supply for the deflector coils in the analyzer, will have to be around 1 part in $10^{6}$, which is also easily feasible. Finally, the whole apparatus will need a bright field emission gun, otherwise there would not be enough intensity available once the monochromator slit is closed down to a width of a few $\mathrm{meV}$, and great attention will have to paid to its probe-forming capabilities. This is because a resolution of a few meV combined with a probe size of a few $\mu \mathrm{m}$ can be attained much more simply by an infrared (IR) light spectrometer, which means that if the electron spectrometer cannot probe significantly smaller sample areas, it may find itself replaced by the IR instrument [38].

\section{Conclusion.}

Instrumentation for electron energy loss spectroscopy has evolved rapidly over the last few years. This has transformed EELS from a technique chiefly distinguished by its versatility [39] into one of the most powerful analytical techniques available. The end of the rapid progress is not yet in sight, and substantial improvements can be expected on several fronts. Chief among these will be efficient energy-filtered imaging and elemental mapping in the STEM mode, imaging spectrometers operating at primary energies up to $1 \mathrm{MeV}$, and ultra-high-resolution spectrometers operating at around $200 \mathrm{keV}$.

\section{References}

[1] HiLlier J. and BAKER R.F., J. Appl. Phys. 15 (1944) 663.

[2] EgERTON R.F., Proc. 39th EMSA meet (G.W. Bailey Ed. Claitor's Publishing, Baton Rouge, 1981) p. 368.

[3] JoHnson D.E. et al. in: Analytical Electron Microscopy 1981 (R.H. Geiss Ed. San Francisco Press, San Francisco, 1981) p. 205.

[4] Shuman H. and Kruit P., Rev. Sci. Inst. 56 (1985) 231.

[5] KRIVANEK O.L., AHN C.C. and KeENEY R.B., Ultramicroscopy 22 (1987) 103.

[6] KRIVANEK O.L., EMSA Bull. 18 (1988) 65.

[7] Castaing R. and Henry L., C.R. Acad. Sci. Paris B255 (1962) 76.

[8] REIMER L., FROMM I. and RENNEKAMP R., Ultramicroscopy 24 (1988) 339.

[9] BRydson R., SAuer H., Engel W., Thomas J.M., Zeitler E., Kosugi N. and Kuroda H., J. Phys. (Condensed Matter) 1 (1989) 797.

[10] BATSON P., these proceedings (1991).

[11] KRIVANEK O.L. et al. Proc. 47th EMSA meet (G.W. Bailey Ed., San Francisco Press, San Francisco, 1989) p. 410.

[12] BoersCh H., Geiger J. and STICKEL W., Z. Phys. 180 (1964) 415.

[13] TERAUCHI M. et al. these proceedings (1991).

[14] KrivaneK O.L., Mory C., TENCÉ M. and Colliex C., these proceedings (1991).

[15] LEAPMAN R.D. and HUNT J., these proceedings (1991).

[16] KUNDMANN M.K. and KRIVANEK O.L., these proceedings (1991).

[17] KrivaneK O.L. and Kundmann M.K., Inst. Phys. Conf. Ser. 98 (1990) 33. 
[18] METHERELl A.J.F., in: Advances in Optical and Electron Microscopy, R. Bauer and V.E. Cosslett Eds. (Academic Press, London, 1971) 4, p. 263.

[19] CURTIS G.H. and SILCOX J., Rev. Sci. Instr. 42 (1971) 630.

[20] HAIDER M., private communication (1990).

[21] KRIVANEK O.L. et al, Electron Microscopy 1990 (L.D. Peachey and D.B. Williams Eds., San Francisco Press, San Francisco, 1990) 2, p. 76.

[22] KRIVANEK O.L., SPENCE J.C.H. and HøIER R., unpublished results.

[23] SENOUSSI S., HENRY L. and CASTAING R., J. de Microsc. 11 (1971) 19.

[24] Zanchi G., PereZ J.-P. and SEVELY J., Optik 43 (1975) 495.

[25] ROSE H. and Pejas W., Optik 54 (1979) 235-250.

[26] LANIO S., Optik 73 (1986) 99.

[27] SHUMAN H. and SOMLYO A.P., in: Analytical Electron Microscopy 1981 (R.H. Geiss Ed., San Francisco Press, San Francisco, 1981) p. 202.

[28] AJika N., Hashimoto H., Yamaguchi K. and ENDOH H., Jap. J. Appl. Phys. 24 (1985) L41.

[29] KRIVANEK O.L. and AHN C.C, Electron Microscopy 1986 (T. Imura, S. Maruse and T. Suzuki Eds., Jap. Soc. Electron Microscopy, Tokyo, Japan, 1986) 1, p. 519.

[30] KRIVANEK O.L. and AHN C.C., in: Intermediate Voltage Microscopy and its Applications in Materials Science, K. Rajan Ed. (Electron Optics Publishing Group, New Jersey, USA, 1987) p. 91.

[31] McMullen D., Rodenburg J.M. and PIKE W.T., Electron Microscopy 1990 (L.D. Peachey and D.B. Williams Eds., San Francisco Press, San Francisco, 1990) 2, p. 104.

[32] SHUMAN H., Ultramicroscopy 5 (1980) 45.

[33] US patent \# 4,851,670.

[34] MOONEY P.M. et al. Electron Microscopy 1990 (L.D. Peachey and D.B. Williams Eds. San Francisco Press, San Francisco, 1990) 1, p. 104.

[35] GUBBENS A.J. and KRIVANEK O.L., to be published.

[36] GeIGER J., Proc. 39th EMSA meet (G.W. Bailey Ed., Claitor's Publishing, Baton Rouge, 1981) p. 182.

[37] US patent application, 1990.

[38] GEIGER J., private communication (1980).

[39] EgERTON R.F., Electron Energy Loss Spectroscopy in a Transmission Electron Microscope (Plenum Press, New York, 1986). 